\title{
Una diversa identità: Carlo Michelstaedter
}

Riassunto: Nella periferica e tranquilla provincia asburgica di Gorizia, lontana dai luoghi di elaborazione e circolazione della raffinata cultura mitteleuropea, un giovane ebreo assimilato, Carlo Michelstaedter, è alla ricerca della sua identità, non sotto l'aspetto dell'appartenenza ad un gruppo nazionale bensì come conquista di una propria individualità, cioè di un'esistenza sottratta alla necessità e alla determinazione, come proposizione di nuovi valori, alternativi a quelli imposti dalla società in cui si trova a vivere.

\section{A different identity: Carlo Michelstaedter}

Abstract: In the peripheral and quiet Habsburg province of Gorizia, far from the places of refined Central European culture, a young assimilated Jew, Carlo Michelstaedter, is in search of his identity, not in the sense of wanting to belong to a nation or specific group, but in the sense of searching his own individuality, an existence beyond necessity and determination, as a proposition of new values, as an alternative to those imposed by the society in which he finds himself living.

Carlo Michelstaedter fu un giovane straordinario: una vita molto breve, la sua (vi pose fine egli stesso con un colpo di rivoltella il 10 ottobre 1910, all'età di 23 anni), ma i cui pochi anni, soprattutto gli estremi, furono fecondissimi di pensiero. Egli è noto soprattutto per La persuasione e la rettorica, un libriccino pubblicato postumo, che è, per quanto atipica nel suo sviluppo e nei suoi esiti, una tesi di laurea (sui concetti di persuasione e di retorica in Platone e in Aristotele), che la morte gli impedì di discutere. È stato definito «filosofo»: giustamente, ma, va precisato, secondo il suo concetto di filosofia, che è quello originario e autentico, di filosofia come arte della vita, di arte di vivere da uomo vero, libero da vincoli, che persegue una vita autonoma fondata su basi razionali, che ha individuato e che cerca di realizzare il vero 'bene'.

Michelstaedter esprime una critica radicale dell'identità intesa come identificazione o nazionale o religiosa o sociale, e ricerca una diversa identità, precisamente l'individualità, concepita come unicità della singola persona. E questa unicità, questa singolarità irripetibile, coincide con quello che egli chiama 
il «persuaso», approdo della sua riflessione, in sostanza la sua risposta ad una domanda che egli si è posto precocemente e perentoriamente, e che è poi quella che da sempre assilla l'uomo che pensa: è possibile riconoscere e conferire un significato assoluto, cioè sottratto alla necessità e alla determinazione, alla propria esistenza? Vediamo.

Michelstaedter è un ebreo di lingua e di cultura italiana, che nasce e vive l'intera sua vita a Gorizia, ${ }^{1}$ ad eccezione dei mesi di studio trascorsi a Firenze, dove tra l'ottobre del 1905 e il giugno del 1909 frequenta l'Istituto di Studi Superiori. Gli abitanti della contea principesca di Gorizia e Gradisca, parte del Küstenland, il Litorale, uno dei quindici Länder dell'Impero austro-ungarico, e di questo estrema propaggine occidentale, erano per due terzi di lingua slovena, ad eccezione del capoluogo, dove la maggioranza era di lingua italiana, con una minoranza slovena ed una, esigua, di lingua tedesca, composta prevalentemente di pensionati della burocrazia imperiale e di personale amministrativo. A Gorizia si parlava inoltre un dialetto veneto, simile al triestino, di cui vi sono tracce nelle lettere di Carlo e anche nella Persuasione. Fra i ceti popolari del capoluogo e nella parte occidentale del contado era diffuso come lingua parlata il dialetto friulano (nella variante goriziana), che in quelli còlti era vezzo farne lingua della poesia: amava verseggiarvi anche il padre di Carlo, Alberto Michelstaedter.

A Gorizia un'ottima conoscenza del tedesco era necessaria per accedere allo Staatsgymnasium, uno degli istituti superiori più prestigiosi dell'Impero, in quanto il tedesco vi fungeva da lingua d'insegnamento. Frequentato da ragazzi di tutto il Litorale, questa rigorosa scuola esclusivamente maschile dal 1850 aveva assunto carattere pluriculturale, poiché agli alunni di nazionalità italiana e slovena era consentito l'apprendimento della madrelingua (per quattro ore settimanali) come lingua straniera, mentre era obbligatorio lo studio delle lingue classiche.

Ervinio Pocar, nativo di Pirano in Istria, uno dei maggiori mediatori e traduttori di letteratura tedesca in Italia, di cinque anni più giovane di Michelstaedter, così ricordava i suoi anni allo Staatsgymnasium goriziano: «Nelle classi eravamo metà italiani, metà sloveni, con un'esigua minoranza di tedeschi, in genere figli di pensionati che dalle città del nord scendevano a stabilirsi a Gorizia per godersi il clima mediterraneo. Noi ragazzi si viveva in un vero caos

1 Prime fonti delle seguenti notizie biografiche e sull'ambiente goriziano sono i profili di Carlo Michelstaedter (anche collettori di quanto emerso dagli studi in merito), anzitutto Un'eterna giovinezza di Sergio Campailla (Venezia, Marsilio, 2019) e Una disperata speranza di Gian Andrea Franchi (Milano-Udine, Mimesis, 2014); si ricorda pure Carlo Michelstaedter di Alessandro Arbo (Pordenone, Edizioni Studio Tesi, 1996). 
linguistico». ${ }^{2}$ Di questo, che peraltro sottende una feconda pluriculturalità, sono prova le poche lettere a Michelstaedter del suo compagno di classe e grande amico Enrico Mreule, originario della provincia di Gorizia, scritte in un italiano singolarissimo e anche scorretto, mescidato di espressioni tedesche e dialettali (ma pure greche e latine). ${ }^{3}$

Gorizia era quindi un crocevia di lingue e di culture diverse, come Trieste, e come Trieste viveva, se pur con intensità indubbiamente minore, crescenti tensioni e contrasti tra la nazionalità italiana e quella slovena (e di entrambe con l'apparato statale austriaco), che si avvertivano latenti anche all'interno dello stesso Staatsgymnasium, come testimonia Biagio Marin, nativo di Grado (allora in territorio austriaco a pochi chilometri dal confine con l'Italia), di tre anni più giovane di Michelstaedter, e anch'egli per alcuni anni alunno dell'istituto goriziano: «Ci rispettavamo. E umano era solitamente il nostro parlare. Ma fuori di là eravamo avversari, e ognuno di noi sapeva che in un prossimo domani ci saremmo trovati di fronte». ${ }^{4}$ Queste tensioni e contrasti nazionali coinvolgevano l'ambiente della famiglia Michelstaedter: il padre Alberto era fieramente irredentista, soprattutto per l'influenza della cognata Carolina Luzzatto, scrittrice, animatrice culturale, fondatrice e direttrice del giornale «Il Corriere Friulano», che dava voce alle istanze della borghesia italiana nazional-liberale.

Secondo il censimento dell'anno 1900, in Gorizia, su una popolazione di poco più di 25.000 abitanti, 248 erano classificati come israeliti, i quali vivevano in buona parte fuori del ghetto, abolito durante l'occupazione francese, e ormai pienamente integrati nella società di maggioranza. L'italiano era la lingua interna della comunità ebraica e quella italiana era la cultura di riferimento, specie della sua borghesia, còlta e culturalmente vivace, e nei suoi membri più noti, come Carolina Luzzato ed Alberto Michelstaedter, di sentimenti irredentistici.

Anche Alberto Michelstaedter si sentiva pienamente integrato e appagato nel contesto sociale, politico e culturale dominante, nella circostante società cattolica. Era uno di quegli ebrei nei quali la definitiva emancipazione e il processo di assimilazione avevano prodotto l'allontanamento dalla stretta osservanza religiosa (quindi il cosiddetto indifferentismo religioso). Scrive la figlia Paula, nei suoi ricordi del fratello Carlo e della famiglia: «Papà era conservativo per le usanze tradizionali ebraiche, ma non era osservante dei riti

2 Citato in G. A. Franchi, Una disperata speranza, cit., p. 21.

3 Riprodotte da Campailla in Dialoghi intorno a Michelstaedter, a cura di id., Gorizia, Biblioteca Statale Isontina, 1987, pp. 24-28.

4 Citato in G. A. Franchi, Una disperata speranza, cit., p. 22. 
né possedeva uno spirito religioso. Anzi era il tipico rappresentante della mentalità materialistica dell’Ottocento». ${ }^{5}$

Di cultura vasta ma non profonda, da autodidatta, Alberto fu un protagonista della cultura locale di lingua italiana, come si evince dalle numerose cariche rivestite a Gorizia (tra cui quella di vicepresidente del Gabinetto di lettura e del Teatro di società); era poi membro attivissimo della Società filologica friulana nonché della Società Dante Alighieri. Si distingueva inoltre come facondo oratore, esperto in discorsi celebrativi e commemorazioni, soprattutto in occasione di anniversari patriottici o letterari italiani (tenne lui nel 1907 l'orazione per la morte di Graziadio Isaia Ascoli). ${ }^{6}$

Alberto si riconosceva in quel piccolo mondo di cultura italiana, i cui modelli letterari erano i due, pur diversi, vati, Carducci e D’Annunzio, anzi i loro versi civili. Questo un passo di un discorso in onore di Carducci da lui tenuto al Gabinetto di lettura di Gorizia la sera del 31 maggio 1901: «Giosuè Carducci, vate d'Italia, come aquila vola su tutti i poeti viventi. Egli che non ha guari sentenziò esser falsa la credenza che la razza latina sia decaduta o moribonda - è una delle più luminose prove della vitalità di questa stirpe». ${ }^{7}$ Come dice giustamente Gian Andrea Franchi, «colpisce la forza dell'identificazione nazionalistica in questo ebreo che parla di "razza latina"». ${ }^{8}$

Dicevamo di tensioni e contrasti tra le diverse nazionalità sempre più evidenti nella Gorizia del tempo. Se coinvolgevano alquanto l'ambiente della famiglia Michelstaedter, Carlo sembra esserne dapprima toccato solo superficialmente. Ci riferiamo ad un episodio accaduto allo Staatsgymnasium goriziano, lo sciopero studentesco dell'ottobre 1904: due classi, di cui quella di Michelstaedter, disertarono le lezioni come forma di protesta contro gli atteggiamenti nazionalistici tedeschi e i metodi didattici autoritari del supplente di storia e geografia, il professor Rudolf Durst. Allo sciopero Carlo partecipò attivamente (di cui risentì la votazione in condotta, senza peraltro incidere sulla sua presentazione alla maturità), lasciandone testimonianza in una caricatura e in due poesie burlesche, dalle quali tuttavia non traspaiono le cause sostanziali dello sciopero. Avvenimento certamente insolito in una scuola asburgica, questo

5 Paula Michelstaedter Winteler, Appunti per una biografia di Carlo Michelstaedter, in appendice a Sergio Campailla, Pensiero e poesia di Carlo Michelstaedter, Bologna, Patron, 1973, p. 147.

6 Fu Ascoli, il celebre glottologo, ebreo goriziano, presto emigrato, nel 1862, nel Regno d'Italia e divenuto infine Senatore del Regno, a dare espressione col termine «Venezia Giulia» all'appartenenza culturale del Litorale all'Italia.

7 G. A. Franchi, Una disperata speranza, cit., p. 32.

8 Ivi, p. 33. 
sciopero rimanda, a modo suo, ad un clima culturale e politico che andava rapidamente deteriorandosi e preannunciava l'ormai vicina esplosione del conflitto tra le diverse nazionalità.

Se i suoi scritti non ne recano tracce significative, Michelstaedter non doveva comunque ignorare questa delicatissima situazione ai confini sud-occidentali dell'Impero. Lo deduciamo da una lettera ch'egli ricevette nel novembre del 1904 da Innsbruck, il cui mittente, rimasto anonimo per la perdita della parte conclusiva della missiva, vi descrive dettagliatamente gravi episodi di intolleranza avvenuti in città: la devastazione dell'inauguranda facoltà italiana di legge dell'Università e di negozi italiani. ${ }^{9}$

Potrebbero trarre in inganno due cartoline postali inviate nel marzo del 1907 da Carlo a Jolanda De Blasi, sua compagna di studi all'Istituto fiorentino e di cui in quei mesi egli si era innamorato. Su di una, che rappresenta il castello di Gorizia, chiosa: «Questo è il castello degli antichi conti di Gorizia. Ora [...] è in mani barbare». ${ }^{10}$ Nell'altra, che raffigura Piazza Grande, e sulla quale indica con una freccia la sua casa, cancella il nome stampato Görz sostituendolo a penna con Gorizia. ${ }^{11}$ Ma qui è un giovane suddito italiano dell'Impero che si rivolge ad una ragazza del Regno, calabrese, e un pizzico d'ingenua e innocua retorica patriottica ci può anche stare, anzi parrebbe inevitabile.

A dissipare ogni dubbio in merito, a mostrare Carlo alieno da ogni sorta di nazionalismo e di irredentismo provvede il poscritto di una lettera scritta alla famiglia il 6 febbraio 1906 da Firenze (quindi anteriore alle cartoline a Jolanda), a proposito di una raccolta di testi patriottici curata dalla zia Carolina, la più risoluta esponente dell'irredentismo isontino:

O Dio dio quasi mi dimenticavo di dirvi l'ineffabile gioia artistica che mi diede quella mer(d)avigliosa raccolta delle perle della stupidità irredenta accompagnate dai paterni commenti. Ma pare che si sia fatto apposta da parte dell'esile raccoglitrice e dei collaboratori di mettere insieme le più grandi stronzaggini. [...] Il sonetto della zia non potrebbe essere più ziocarolinesco [...] però mi dà pensiero per lo stato della sua mente, è davvero da manicomio, non mi fa più ridere, mi fa piangere, dio che colmo. ${ }^{12}$

A far comunque e definitivamente testo sono alcuni versi vergati nel 1910 (e qui sta il paradosso, «su richiesta di alcune signore filoitaliane, per un numero unico

9 Lettera riprodotta da Campailla in Dialoghi intorno a Michelstaedter, cit., pp. 8-13.

10 Carlo Michelstaedter, Epistolario, a cura di Sergio Campailla, Milano, Adelphi, 1983, p. 195, nota 2.

11 Ibid.

12 Ivi, p. 100. 
patriottico, dove poi non furono pubblicati», come informa Campailla): ${ }^{13}$ "Non è la patria / il comodo giaciglio / per la cura e la noia e la stanchezza; / ma nel suo petto, ma pel suo periglio / chi ne voglia parlar / deve crearla», ${ }^{14}$ nei quali si coglie una concezione ormai chiara e salda dell'individuo, non come identità data (nazionale o religiosa o sociale) ma come singolarità irripetibile che ciascuno deve crearsi da sé.

La famiglia di Alberto Michelstaedter, come si è detto, era ormai laicizzata e pienamente integrata nella società locale, e della religione ebraica conservava aspetti prevalentemente formali. Carlo mostra estraneità se non insofferenza e anche repulsione nei confronti dell'ambiente ebraico osservante, cui appartiene il giovane con cui la sorella Paula vive una sofferta vicenda amorosa tra il 1905 e il 1906. Così a Paula da Firenze a fine gennaio 1906:

non si può farsi illusioni contro un ideale così forte così vivo come la fede, che ha in lui le radici più profonde congenite (perché egli non è un'eccezione. Ma in quell'ambiente ho conosciuto 5 sei giovani, tutti gli ebrei che vengono all'istituto cioè meno uno o due che hanno le stesse idee, lo stesso fervore, che studiano tutti al collegio rabbinico) [...]. Mi fa l'impressione di una aberrazione generale. Sono gente che non vivono nel nostro mondo, che non possono partecipare che materialmente alla nostra vita; ${ }^{15}$

e nella primavera del 1906: «Io l'ambiente l'ho conosciuto [...] e ti so dire che è stato un bene per te non entrarci. Sono buona gente ma di una bontà passiva e sul punto di religione intransigente a costo di qualunque cosa. Mi fa l'impressione come di vederti liberata da un incubo, di vederti uscire da una camera chiusa oscura al sole chiaro allegro». ${ }^{16}$

La famiglia era comunque inserita in una rete parentale e amicale estesa oltre Gorizia, a Trieste, Venezia, Vicenza, Padova, Ferrara, Firenze, e che raggiungeva anche Vienna e New York. Carlo frequenta senza entusiasmo alcune di queste famiglie ebree, ad esempio i fiorentini Della Pergola. Così ancora a Paula da Firenze sempre a fine gennaio 1906: «Ieri c'era un ballo per Purim, io sono stato invitato da un giovane del comitato che è mio amico. Veramente non volevo andarvi per non trovarmi in un ambiente sionistico, poi per le istanze che m'hanno fatto e specialmente in casa dai Pergola, e perché anche il sig. Cesare era del comitato dovetti andarci». ${ }^{17}$

13 Carlo Michelstaedter, Poesie, a cura di Sergio Campailla, Milano, Adelphi, 1987, p. 108.

14 Ivi, p. 76.

15 C. Michelstaedter, Epistolario, cit., p. 91.

16 Ivi, p. 119.

17 Ivi, p. 111. 
Vi è in queste righe un accenno al sionismo, allora nel suo slancio iniziale (Herzl aveva pubblicato nel 1896 Lo stato degli ebrei, in cui vedeva l'identità nazionale ebraica doversi necessariamente tradurre nella costruzione di uno stato degli ebrei, ed era morto soltanto da due anni), nei cui confronti l'avversione più violenta veniva, oltreché dagli ambienti più rigorosamente religiosi, dalla borghesia ebraica assimilata, cui l'Impero aveva garantito emancipazione economica e sicurezza sociale. Il rifiuto del sionismo da parte di Michelstaedter conferma il suo rifiuto di ogni forma di identificazione, in questo caso nazional-religiosa.

Torniamo al padre Alberto. Dalle testimonianze, di lui e su di lui, emerge come profondamente radicato nell'ordine borghese e pienamente conforme alle regole sociali. Scrive, ad esempio, nella lettera a Carlo nota come 'sermone paterno', sorta di viatico etico-sociale per il figlio in procinto di affrontare da solo nell'ottobre del 1905 l'esperienza fiorentina: «tuo padre [...] ha fatto base della propria esistenza l'onore, sua legge suprema l'onesto lavoro, sua religione il dovere». ${ }^{18}$ Potremmo dire, richiamando proprio il milieu culturale ebraico, che Alberto di fatto ispirava la sua condotta nei confronti dell'autorità costituita a quanto insegnano a più riprese i maestri del Talmud («la legge del luogo in cui risiedi è legge»), e che questa rappresentava invece il bersaglio principale della radicale critica filosofica e sociale di Carlo, il quale, al pensiero e al costume vigente («Essi [«gli uomini»] dicono: "Non siamo né i primi né gli ultimi a questo mondo, e, poiché bisogna vivere, conviene adattarsi a quello che si trova, che d'altronde non potremmo cambiare") ${ }^{19}$ oppone «la via della persuasione», che «non ha che questa indicazione: non adattarti alla sufficienza di ciò che t'è dato». ${ }^{20}$

18 In Dialoghi intorno a Michelstaedter, cit., p. 13. Aveva scritto precedentemente: «Stai per lasciarci e te ne vai giovialmente nella radiosa Firenze a fare un bagno d'arte e di lettere, ad ornarti lo spirito delle nozioni più attraenti e poetiche. [...] spero che la tua coscienza t'avvertirà sempre che non vai a godere soltanto, che hai doveri da compiere [...] ogni nostra azione dev'essere retta dal criterio che prima d'ogni altra cosa dobbiamo compiere il nostro dovere. - Il dovere è il faro, la guida che "mena dritto altrui per ogni calle", il culto più degno dell'uomo superiore [...]. Stai per lasciarci, per essere privato della tua guida naturale, sarà un grande avvenimento per te il sentirti da un momento all'altro appoggiato a te solo, i miei consigli dunque derivati dall'affetto intenso e dall'esperienza sono il tuo viatico necessario ed io ho abbastanza fiducia in te per calcolare che nell'educazione ricevuta, nei radicati principi di rettitudine, nell'istintivo senso del bene attingerai i freni che sostituiranno nella tua nuova esistenza la direzione nostra» (ivi, p. 10).

19 Carlo Michelstaedter, La persuasione e la rettorica, a cura di Sergio Campailla, Milano, Adelphi, 1982, p. 73.

20 Ivi, p. 104. 
Alberto non avrebbe quindi potuto comprendere il figlio, per il quale man mano che maturava in lui il 'filosofo' egli diventava una figura non solo estranea ma paradigmaticamente antitetica: il «grosso signore», appunto, il borghese, l'uomo che «si adatta ragionevolmente», a ragion veduta, protagonista del dialogo che apre il terzo capitolo della seconda parte della Persuasione, intitolato $L$ a rettorica nella vita, ${ }^{21}$ dialogo che si conclude con un emblematico riferimento alla professione del padre, il quale, dapprima cambiavalute, si dedicò poi anche, e quindi esclusivamente, all'attività assicurativa, divenendo il Direttore dell'Agenzia delle Assicurazioni Generali di Trieste a Gorizia. ${ }^{22}$

Con la sua esperienza familiare si confronta, e certo se ne alimenta, anche la maturazione filosofica di Carlo, l'elaborazione del suo pensiero. Ed è la divergenza, sempre più netta, incomponibile e consapevole, sulla concezione della vita a causare la sua progressiva estraneazione dall'ambiente familiare, sempre più patito, anche se ricco di affettività («sai [...] come siamo noi in famiglia uniti quasi in un unico punto caldo», ${ }^{23}$ scrive Carlo all'amico Gaetano Chiavacci, il 26 febbraio 1909, annunciandogli la morte del fratello maggiore Gino).

Come sappiamo, le matrici di pensiero sono spesso sotterranee, agiscono sotto la soglia della coscienza. Nella radicalità con cui Carlo contesta i valori, le mete e i modelli di condotta dominanti opponendovi valori nuovi e alternativi, potremmo vedere una peculiarità intellettuale ebraica, la propensione a rompere gli schemi dati, a disgregare certezze consolidate. Egli rifiuta infatti ogni identità data, «costituita», sia essa nazionale o religiosa o sociale, affermando la necessità vitale di una diversa identità, che ciascuno deve crearsi da sé: «L'unica cosa che vale è il "valore individuale”. [...] questo deve ognuno aver il coraggio di far diventare "realtà"»; ${ }^{24}$ "Chi vuole fortemente la sua vita [...] deve crear sé stesso per avere il valore individuale». ${ }^{25}$

«Individuo», quindi, non come numero della specie, segmento infinitesimale e anonimo di quell'unica «corda tesa» nel tempo - per usare un'espressione

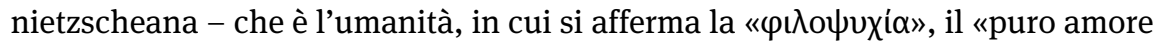
della vita», ${ }^{26}$ la cieca brama di vita, ma come singolarità irrepetibile che coincide

21 Ivi, p. 137.

22 Dice ad un certo punto il «grosso signore»: «bisogna aver coscienza d'aver fatto il proprio dovere. Oh questo sì, sul dovere non si transige» (ivi, p. 138). Vedi (sopra e alla nota 18) quanto Alberto Michelstaedter aveva detto sul dovere nel 'sermone paterno' a Carlo.

23 C. Michelstaedter, Epistolario, cit., p. 352.

24 C. Michelstaedter, Opere, a cura di Gaetano Chiavacci, Firenze, Sansoni, 1958, p. 700.

25 C. Michelstaedter, La persuasione e la rettorica, cit., p. 72.

26 C. Michelstaedter, Opere, cit., p. 715. 


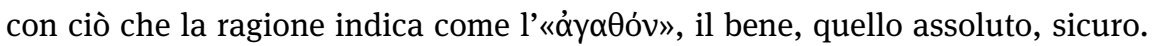
Che per Michelstaedter, e per il suo «persuaso», consiste nel possedere pienamente la propria vita, in quanto emancipata da ogni necessità e determinazione, spezzando le catene con cui la « l'uomo, e nella quale ha origine la costituzione del sapere dato, costituto, la «rettorica» appunto. E insieme il bene consiste nel «far beneficio», cioè nell'agire secondo giustizia, che è un'idea-limite, una mèta, alla quale, pur consapevole della sua inattingibilità, vuole tendere chi ha imboccato la via della «persuasione»: certo l'individuo così inteso è qualcosa di impensato e intollerabile per la società, nella quale «non vive l'individuo, ma vive in tanti individui la società stessa». ${ }^{27}$ In questo consiste l'identità vera, da perseguire, per Carlo Michelstaedter, al quale tuttavia la morte prematura impedì di verificarne la possibilità.

27 Ivi, pp. 100-101. 
